\title{
Introduction to Baby Café USA
}

Lucia Jenkins, RN, IBCLC, RLC

Baby Café USA, a nonprofit organization affiliated with Baby Café Charitable Trust of the U.K., was established in February 2012 by Lucia Jenkins, RN, IBCLC, RLC, to promote the development of U.S. Baby Cafés, specifically targeting underserved areas with low breastfeeding rates. Baby Cafés are drop-in, free-of-charge breastfeeding support centers that operate by standards and guidelines set by Baby Café USA jointly with Baby Café UK.

Baby Cafés provide crucial support to thousands of breastfeeding mothers around the world and, in addition, gather demographic and statistical data on attending mothers. The U.K. organization began in 2000, and currently supports more than 120 successful Cafés worldwide, individually funded by a wide range of local community, private, and national grants. Baby Café USA is the first international affiliate organization to be established outside of the U.K. and has developed specific U.S. requirements, which include facilitation by an International Board Certified Lactation Consultant (IBCLC).

Ms. Jenkins introduced the Baby Café concept to Hallmark Health System (HHS) and Melrose-Wakefield Hospital in 2006. Melrose-Wakefield Hospital opened the first U.S. Baby Café that year and has opened two additional Women, Infants, and Children (WIC)based Cafés, all of which HHS continues to fund. Baby Cafés are baby friendly, meet at least once a week, are accessible by public transportation, and offer peer-support counseling as well as professional-level lactation care. Baby Cafés fulfill Step 10 of Baby-Friendly Hospital Initiative requirements and are currently open in Massachusetts, Texas, Florida, Minnesota, Alabama, Michigan, Pennsylvania, South Carolina, North Dakota, and New York, with more in the development process.

In addition to tracking exclusivity rates of mothers at Café meetings, Ms. Jenkins has developed statistical gathering tools and a call-back survey to track duration and other factors influencing a mother's breastfeeding experience. This data is valuable to evaluate each Café's impact on community breastfeeding rates. Four years of data from the HHS Cafés are in the final stages of editing and will be submitted for publication in the next few months. Baby Café USA will continue to collect and evaluate all data from U.S. Cafés using standardized statistical gathering tools.

The preliminary results from HHS Cafés demonstrate, for example, that the café model positively impacts the duration goals mothers have set for themselves prenatally: approximately $82 \%$ reach their goals; $74 \%$ of café moms increase their duration goals; and $71 \%$ reach the American Academy of Pediatrics (AAP) goals of breastfeeding for 1 year.

For more information on finding a Baby Café or information on opening a Baby Café in your community, please visit www.babycafeusa.org.

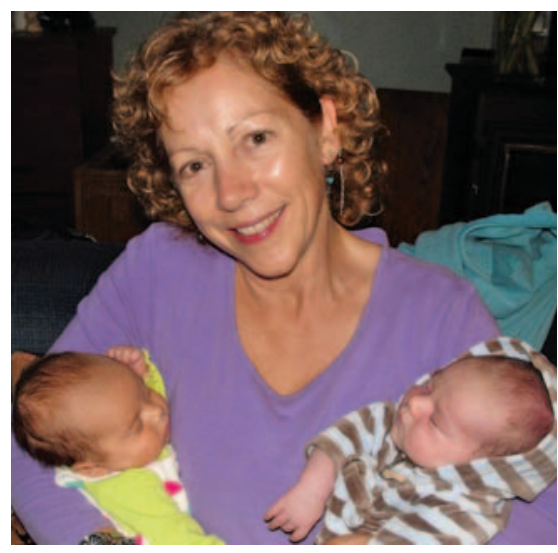

Lucia Jenkins, RN, IBCLC, RLC, is an IBCLC with 19 years of experience, and is the originator and executive director of Baby Café USA. She is a staff IBCLC at Melrose-Wakefield Hospital, has a private lactation practice, and is a board member of the Massachusetts Breastfeeding Coalition. She is the principal facilitator of the first USA Baby Café, and the first WIC Baby Café, both funded by HHS. Lucia has lectured at various conferences and state coalitions including Massachusetts Department of Public Health (DPH), International Lactation Consultant Association (ILCA) 2009, and United States Breastfeeding Committee (USBC) 2012 about Baby Cafés and the process of starting them.

1. Lucia@babycafeusa.org, Executive Director, Baby Café USA, Inc.,

Wakefield, MA, Nonprofit Affiliate of Baby Café Charitable Trust, UK 